International Journal of Current Advanced Research

ISSN: O: 2319-6475, ISSN: P: 2319 - 6505, Impact Factor: SJIF: 5.995

Available Online at www.journalijcar.org

Volume 6; Issue 3; March 2017; Page No. 2341-2346

DOI: http://dx.doi.org/10.24327/ijcar.2017.2346.0005

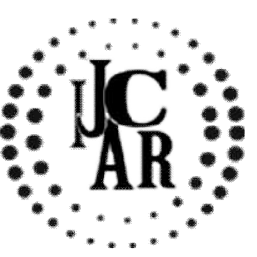

Research Article

\title{
A REVIEW ON SINGULAR PERTURBED DELAY DIFFERENTIAL EQUATIONS
}

\author{
*Mandeep Kaur and Geeta Arora
}

Lovely Professional University, Punjab

\section{A R T I C L E I N F O}

\section{Article History:}

Received $18^{\text {th }}$ December, 2016

Received in revised form $16^{\text {th }}$ January, 2017

Accepted $26^{\text {th }}$ February, 2017

Published online $28^{\text {th }}$ March, 2017

\section{Key words:}

Perturbed differential equations, delay, collocation method, Finite Element Method.

\begin{abstract}
A B S T R A C T
In this paper, existing available literature on numerical solution of singularly perturbed and singular perturbed delay differential equations is reviewed. These equations arises in mathematical modeling of variational problems in control theory,physical and biological phenomena like optically bistable devices, description of human pupil reflex and a variety of models for physiological processes or diseases. The purpose of this work is to investigate the classof singular type problems that are solved till now using different numerical methods. The objective is to motivate the researchers to develop new methods for calculating efficient solution of such problems. There is a lot of work reported in literature to solve these typesof equations both numerically and analytically. This paper limits its coverage to the work done by numerous researchers between 2002 and 2017.
\end{abstract}

Copyright $@ 2017$ Mandeep Kaur and Geeta Arora. This is an open access article distributed under the Creative Commons Attribution License, which permits unrestricted use, distribution, and reproduction in any medium, provided the original work is properly cited.

\section{INTRODUCTION}

The peculiarity of detecting the relation between causes and effect emerge when the cause is small and effect is large. In theory of perturbation formathematics and physical systems, the study of this relation got significance amount of attention in past and recent years. The work on finding the solution of singular perturbed differential equations (SPDE) started in year 1968. Various studies and surveys have been conducted by researchers on the development in this area. A survey on different asymptotic and numerical methods for solving singular perturbation problems done by Kadalbajoo and Reddy[1]. In 2002, Kadalbajoo and Patidar [2] presented another surveyonthework done by numerous researchers in the area of singular perturbation from 1984 to 2000. Kadalbajoo and Patidar [3] presented are view of singularly perturbed partial differential equations in 2003.In [4] Kadalbajoo and Vikasgave brief survey on the computational techniques used to solve different classes of singular perturbation problems. In 2013, Sharma et al. [5] presented a review on singular perturbed differential equations with turning point and interior layers. From this discussion it can be concluded that this area has developed much in the past century. So it motivates us to write a review paper on work done by researchers for solving these equations.

In this survey we restrict our study mainly on singular perturbed delay differential equation which is a type of

*Corresponding author: Mandeep Kaur,

Lovely Professional University, Punjab singular perturbed differential equations and very useful in wide range of applications in real life.In this survey we have given very brief review for the work done on singular perturbed differential equations and we have given detail review of singular perturbed delay differential equations.

The paper is organized as follow: In section two, singular perturbed differential equations are introduced and applications of these equations discussed. In section three, survey of numerical solution on singular perturbed differential equation is given. In section four, singular perturbed delay differential equations are introduced and applications of these equations are given. In section five, methods used for solving singular perturbed delay differential equations are presented. Finally the paper is concluded with further scope of work in this area followed by references.

\section{Introduction to singularly perturbed differential equations}

A singularly perturbed differential equation is an ordinary differential equation in which the highest order derivative is multiplied by a small parameter known as perturbation parameter. The solution of singularly perturbed differential equations (SPDE) varies rapidly in the regions called as layers which may be apparent in the solution or its derivatives and often appear at the boundary of the domain. The study of solution of these equations is of great significance due to the formation of sharp boundary or interior layers when the perturbation parameter approaches to zero.

In real life, many problems in science and engineering, control theory, elasticity, fluid mechanics, biosciences are modeled by singular perturbed differential equations, for instant, in red cell system [6], variational problems in control 
theory $[7,8,9]$, describing the motion of the sunflower [10], signal transmission [11] and, depolarization in the Stein model [12], thermo elasticity [13], Optics and physiology [14] etc.

\section{Work progress towards solution of SPDE}

The general form of SPDE of second order solved is

$$
\begin{gathered}
\varepsilon u^{\prime \prime}(r)+f\left(r, u, u^{\prime}\right)=0 \text { subject to condition } u(a) \\
=A \text { and } u(b)=B \text {. }
\end{gathered}
$$

To solve the perturbed differential equation following techniques has been used by the researchers:

Spline based methods: The idea behind spline is fitting of piecewise continuous polynomial passing through given points. A function $\mathrm{s}(\mathrm{x})$ is a spline of degree $\mathrm{k}$ if it is $\mathrm{k}^{\text {th }}$ degree polynomial $\mathrm{p}(\mathrm{x})$ and its first $\mathrm{k}-1$ derivatives are also continuous everywhere in domain $\left[\mathrm{x}_{0}, \mathrm{x}_{\mathrm{n}}\right]$. There is remarkable progress in the theory on numerical methods and applications for solution of SPDE. The spline based methods are easy and ready for computer implementation.

Following is the work reported in literature on spline based method: SPDE of different order extensively studied in literature because of their multiple applications [6-14] by the researchers. Aziz and Khan [15] in 2002 proposed the solution of second order singularly perturbed boundary value problem by using cubic spline in compression and interpolatory condition. The considered equation was of form

$$
\begin{gathered}
\varepsilon y^{\prime \prime}=p(x) y^{\prime}+q(x) y+r(x), \quad y(a)=\alpha_{0} \text { and } y(b) \\
=\alpha_{1}
\end{gathered}
$$

Depending on the two parameters involved in method, scheme found to have second and fourth order of convergence. In 2012, Jha [16] used finite difference method for nonlinear singularly perturbed singular boundary value problem of second order using non polynomial spline finite difference method. Author claimed that method is fourth order convergent and is easy to implement. He considered two test examples to check efficiency of the scheme and calculate the root mean square error. In 2013, El-Salam [17] used a second and fourth order convergent parametric spline method for second order singularly perturbed boundary-value problem. He has shown that the order of convergence depends on the choice of two parameters. Akram and Naheed [18] in 2013 solved fourth order singularly perturbed boundary value problem using septic spline. In this paper spline of septic degree are substituted in the equation to obtain numerical solution by applying the properties of continuity of derivatives of second, fourth and sixth order. Author proved the method to be of fourth order convergent and having better results as compared to the existing solution.

Remarks: In spline based methods, the domain is divided into subintervals by selecting uniform partition and spline approximation methods such as collocation, finite element etc. were used to approximate the solution.Spline based methods provides accurate results but one needs skill to handle the calculations.

Transformation Methods: In mathematics transformation is an operation that converts one function into another. For example Laplace is the integral transform used to find the solution of differential equations. Following transformation methods are reported in literature to find the solution of
SPDE: Dogan et al. [19] in 2012 numerically treated singularly perturbed two point boundary value problem using differential transformation method. This method is an iterative method for procuring analytical Taylor series solutions of differential equations and it can be used to solve both linear and nonlinear initial value problems. In 2013, Mishra and Saini [20] discussed the numerical solution of singularly perturbed two-point boundary value problem via LiouvilleGreen Transform. It is simple and easy to implement analytical method for obtaining solution of differential equations.

Remarks: Both transformation methods presented analytical solution of SPDE as a function of $\mathrm{x}$ instead of giving approximate value of solution at selected points. The solution of equation could be obtained at any point in domain.

Methods using B-spline as basis function: A spline function that has minimal support with respect to the given degree, smoothness and domain partition is called B-spline. This basis function has been used by various researchers to solve linear and nonlinear differential equations. Gupta et al. [21] in 2011 applied cubic B-Spline scheme to find numerical solution of system of singularly perturbed boundary value problems. In 2015 Mishra and Saini [22] discussed quartic B-Spline basis function for solving singular singularly perturbed third-order boundary value problems. In this paper basis of quartic Bspline is derived and matrix form of the system is solved by using MATLAB. The efficiency of the method is presented by implementing on test examples and absolute error was discussed.

Remarks: In above mentioned papers mathematicians used BSpline basis function of order two and three respectively. The discussed technique is easy to apply and provides better approximate solution due to compactly support properties of $\mathrm{B}-$ Spline basis functions.

\section{Introduction to singularly perturbed delay differential equations}

Singularly perturbed delay differential equation is an equation in which evolution of system at a certain time depends on the rate at an earlier time. The delay in process arises due to requirement of definite time to sense the instruction and react to it. The periodic oscillation of breathing frequency under constant conditions shown by some person is an example of delay equation. This delay is caused by cardiac insufficiency in the physiological circuit controlling the carbon dioxide level in blood [23]. For more applications of delay differential equations in Biosciences refer [23]. The delay differential equation in which the highest derivative is multiplied by perturbation parameter is known as perturbed delay differential equation. The delay differential equation can be classified as retarded delay differential equation and neutral differential equation.The applications of delay differential equations arises in modeling of neural variable,variational problems in control theory [24,25], description of human pupil reflex [26], physical and biological phenomena like optically bistable devices [27] and in numerical modeling in biosciences[28], HIV infection [29]etc.

Expansion in area of finding solution of singular perturbed delay differential equations

A general form of SPDDE is 


$$
\begin{gathered}
\varepsilon y^{\prime \prime}(x)+a(x) y^{\prime}(x-\delta)+b(x) y(x-\delta)+c(x) y(x) \\
=f(x), \quad 0<x<l
\end{gathered}
$$

subject to the conditions $\mathrm{y}(\mathrm{x})=\emptyset(x)$ on $-\delta \leq x<0$.

A lot of work has bee reported in literature for finding the approximate solution of these equations. Following are the techniques used for finding solution of these equations:

\section{Spline and B-spline based methods}

A tremendous advancement has been reported in past decant in the theory of Spline and B-spline based methods for finding numerical solution of differential equations.An optimized Bspline method to solve singularly perturbed differential difference equation with delay as well as advancewas proposed by Sharma [30]. The considered equation was:

$$
\begin{aligned}
& \varepsilon u^{\prime \prime}(x)+a(x) u^{\prime}(x)+a(x) u(x-\delta)+\omega(x) u(x) \\
& +\beta(x) u(x+\eta)=f(x) \quad \forall x \in \Omega
\end{aligned}
$$

with the conditions $u(x)=\varnothing(x)$ on $-\delta \leq x 0$ and $u(x)=$ $\gamma(x)$ on $1 \leq x \leq 1+\eta$

In this paper author used collocation method to find the solution of the problem by using shishkin mesh strategy. It was claimed that the proposed method was of second order of convergence.

In 2012, Kumar [31] treated singularly perturbed delay differential equation of second order using B-spline collocation method.In this article the author has selected piecewise uniform mesh known as shishkin mesh which is adequate to handle singularly perturbed problems. The mesh is constructed in such a way that more mesh points are created in the boundary layer region than outside these regions. The SPDE of second order was treated by the cubic B-spline scheme and the solution obtained by solving tri-diagonal matrix. Author discussed the uniform convergence of the method and to exhibit the coherence of the method two test examples were considered. As the exact solution of the considered problems is not known so the maximum absolute nodal error is estimated by $E_{\epsilon}^{N}=\max \left|y_{i}^{N}-y_{2 i}^{2 N}\right|, 0 \leq i \leq N$. Author represented the results by maximum absolute error obtained by using uniform and shishkin mesh. It was observed that the error with the shishkin mesh was less as compare to the uniform mesh as value of $\mathrm{N}$ increase.

Chakravarthy et al. [32] solved these equations by using cubic spline in compression.In this paper author considered the SPDDE of second order and with large delay. Uniform mesh was selected to partition the domain and method based on cubic spline in compression was used to approximate the solution of the problem.

The equation is of form: $\varepsilon y^{\prime \prime}(x)+a(x) y^{\prime}(x)+b(x) y(x-$ 1) $=f(x) \quad 0 \leq x \leq 2$

with $y(0)=\varnothing(x):-1 \leq x \leq 0$ and $y(2)=\beta$. Author claimed that the presented schemes provide accurate results for the linear SPDDE with large delay.

To demonstrate the efficiency of method author applied the discussed technique on five test examples and presented results in tables for different values of $\mathrm{N}$ (total number of partition points). Maximum absolute error was calculated with double mesh principle. It was observed that the absolute error decreases with increase in value of $\mathrm{N}$.
Remarks: Spline based methods provide more accurate results for SPDDE with small delay, large delay and for the SPDDE with delay as well as advance. These methods are easy to apply due to properties of spline functions.

\section{Exponential collocation method}

In 2013, YuzbagjandSezer [33] used an exponential collocation method for finding solution of second order SPDDE. Author discussed the solution of the problem by considering the exponential basis set $\left\{1, \mathrm{e}^{-\mathrm{x}}, \mathrm{e}^{-2 \mathrm{x}}, \ldots, \mathrm{e}^{-\mathrm{Nx}}\right\}$ and solution was of the form $\mathrm{y}(\mathrm{x})=\sum_{n=0}^{N} a_{n} e^{-n x}$. The boundary $0 \leq x \leq b$ was divided with constant mesh length $\mathrm{b} / \mathrm{N}$. The approximate solution and its derivatives presented in matrix form and substituted in differential equation. Based on the residual function $R_{N}(x)=L\left[y_{N}(x)\right]-g(x)$, error function was determined and corrected exponential solution was presented. Four test examples were solved to demonstrate the efficiency of the method. Absolute error in all the examples was calculated for different values of $\mathrm{N}$.

\section{Computational Techniques}

In 2013, File et al. [34] presented a computational method to solve these equations with negative shift whose solution has boundary layer. In this scheme author reduced the second order SPDDE to first order equation and then employed numerical integration and interpolations. Author claim that the available asymptotic expansion methods for solving singular perturbed problems are difficult to apply as it is not easy to find appropriate asymptotic expansions in the inner and outer regions and matching of the coefficients of the inner and outer solution expansions is also a process that need skills. The proposed method overcame above problems. The left ended and right ended problem was treated separately. Taylor series expansion of $\mathrm{y}(\mathrm{x})$ in neighborhood of $\mathrm{x}=0$ and $\mathrm{x}=1$ is used to reduce second order singularly perturbed delay differential equationin first order delay differential equation. The interval $[0,1]$ is divided in equal subparts of constant length $1 / \mathrm{N}$. The new equation integrated over an interval and solved by Trapezoidal Rule and Taylor series. Author discussed the discrete invariant imbedding algorithm to solve three term recurrence relation. Method employed on four examples and the result of method compared by the exact in two examples.

In 2015, D. Kumara Swamy et al. [35] proposed computational method for singularly perturbed delay differential equation of second order with twin layers or oscillatory behavior. Layer or oscillation behavior of the delay differential equation discussed depending on sign of $(a(x)+$ $\mathrm{b}(\mathrm{x})$ ). The layer behavior of the solution diminish as the delay increases and the solution exhibit oscillation behavior. The interval $[0,1]$ is divided into even number of sub intervals with constant mesh length .The second order delay differential equation is transformed into an equivalent first order neutral type delay differential equation. The new equation integrated on the interval $[0,0.5]$ and $[0.5,1]$ using Trapezoidal Rule.

In 2013, Devendra Kumar [36] solved boundary-value problem for a singularly perturbed delay differential equation with a small parameter multiplying to the second derivative and containing negative shift in the first derivative term.

Taylor's series expansions of the terms that involve delay are used and central difference formula used for first order 
derivative term. The method employed on a test example to check the result.

Remarks: In above discussed methods original SPDDE reduced to first order equivalent equation and integrated numerically to obtain the solution.

\section{Other techniques Used}

Asymptotics -fitted method: In 2012, Andargieet et al. [37] proposed an Asymptotics -fitted method for solving singularly perturbed delay differential two point boundary value problem of second order. The equation of form:

$\varepsilon y^{\prime \prime}(x)+a(x) y^{\prime}(x-\delta)+b(x) y(x)=f(x) \quad 0 \leq x \leq 1$ with $y(0)=\alpha:-\delta \leq x \leq 0$ and $y(1)=\beta$ was considered. Taylor's expansion is used to approximate the term containing negative shift. In the new equation a fitted parameter on the highest order derivative is introduced and its value is determined by using theory of Singular Perturbation. Uniform mesh was selected and the interval $[0,1]$ is divided into $\mathrm{N}$ equal parts with constant mesh length. Thomas Algorithm was discussed to solve three term recurrence relation. The proposed method was implemented on five test examples to demonstrate the efficiency of the method. Out of the 5 examples 3 examples were linear and other 2 nonlinear. On nonlinear examples quasi- linearization method was used. The delay parameter $\delta$ is chosen to reduce the coefficient of second derivative of the modified problem. The absolute error in all the five examples has been discussed. From the result it was observed that with the increase in $\mathrm{N}$ the absolute error decrease. differential equation for the inner region is solved by using second order classical finite difference scheme. To solve the outer region problem uniform mesh with constant length has been considered and classical central finite difference scheme is used. Finally to obtain the solution of original problem the two solution-inner region and outer region are combined.

A hybrid initial value method: In 2016 Subburayan [40] used a hybrid initial value method with shishkin mesh strategy to SPDDE of second order with discontinuous coefficient of derivative term. In the presented scheme second order differential equation reduced in three singular perturbed differential equations and one regular differential equation with delay parameter. Hybrid finite difference scheme is used to solve the three singular perturbed differential equations and to solve the delay differential equation improved Euler method was employed. Author claimed the method is second order convergent.

A fourth order finite difference scheme presented by Gemechis File et al. [41] in 2017 for numerical solution of SPDDE. The authors considered Reaction-Diffusion Equation

$$
\varepsilon^{2} y^{\prime \prime}(x)+a(x) y(x-\delta)+b(x) y(x)=f(x) \quad 0 \leq x
$$

with $y(x)=\emptyset(x)-\delta \leq x \leq 0$ and $y(1)=\beta$ and uniform mesh with constant mesh length is considered. A fourth order finite difference scheme is derived by using Taylor series expansion and the resultant tri-diagonal system solved by the method of Discrete Invariant Imbedding Algorithm. Stability analysis of the above scheme was carried out and found that the method is of fourth order uniformly convergent.

\begin{tabular}{|c|c|c|}
\hline Technique Used & SPDDE & Method \\
\hline & $\begin{array}{l}\text { 1. } \varepsilon u^{\prime \prime}(x)+a(x) u^{\prime}(x)+a(x) u(x-\delta)+\omega(x) u(x)+ \\
\beta(x) u(x+\eta)=f(x)\end{array}$ & $\begin{array}{l}\text { Cubic B-spline collocation method } \\
\text { withshishkin mesh [30]. }\end{array}$ \\
\hline Spline and B-spline & $\begin{array}{l}2 \varepsilon y^{\prime \prime}(x)+a(x) y^{\prime}(x-\delta)+b(x) y(x-\delta)+ \\
c(x) y(x)=f(x)\end{array}$ & $\begin{array}{c}\text { Cubic B-spline collocation method } \\
\text { withshishkin mesh[31]. }\end{array}$ \\
\hline Exponential Basis & $\begin{aligned} \text { 3. } \varepsilon y^{\prime \prime}(x)+a(x) y^{\prime}(x)+b(x) y(x-1) & =f(x) \\
\varepsilon y^{\prime \prime}(x)+p(x) y^{\prime}(x-\delta)+r(x) y(x) & =g(x)\end{aligned}$ & $\begin{array}{l}\text { Cubic B-spline in compression with uniform mesh [32]. } \\
\text { Exponential collocation method with uniform mesh [33]. }\end{array}$ \\
\hline $\begin{array}{l}\text { Computational } \\
\text { Techniques }\end{array}$ & $\begin{array}{l}\varepsilon y^{\prime \prime}(x)+a(x) y(x-\delta)+b(x) y(x)=f(x) \\
\varepsilon y^{\prime \prime}(x)+a(x) y^{\prime}(x-\delta)+b(x) y(x)=f(x) \\
\varepsilon y^{\prime \prime}(x)+p(x) y^{\prime}(x-\delta)-q(x) y(x)=f(x)\end{array}$ & $\begin{array}{l}\text { Equation reduced to asymptotic equivalent equation using Taylor's } \\
\text { series with uniform mesh [34]. } \\
\text { Taylor's series expansion used in neighborhood of } x=0 \text { or } x=1 \text { in case } \\
\text { of Boundary layer on left or right side respectively with uniform mesh } \\
\text { [35]. }\end{array}$ \\
\hline Other techniques Used & $\begin{array}{ll}\text { 1. } & \varepsilon y^{\prime \prime}(x)+a(x) y^{\prime}(x-\delta)+b(x) y(x)=f(x) \\
2 . & -\varepsilon^{2}\left(u^{\varepsilon}\right) "(x)+a(x) u^{\varepsilon}(x)+b(x) u^{\varepsilon}(x-1)=f(x) \\
3 . & \varepsilon y^{\prime \prime}(x)+a(x) y^{\prime}(x-\delta)+b(x) y(x)=f(x) \\
4 . & -\varepsilon u^{\prime \prime}(x)+a(x) u^{\prime}(x)+b(x) u(x-1)=f(x) \\
5 . & \varepsilon^{2} y^{\prime \prime}(x)+a(x) y(x-\delta)+b(x) y(x)=f(x) \\
6 . & \varepsilon y^{\prime \prime}(x)+p(x) y^{\prime}(x-\delta)+q(x) y(x)=r(x)\end{array}$ & $\begin{array}{l}\text { Taylor's series with uniform mesh [36]. } \\
\text { Asymptotics -fitted method, by introducing a fitting factor with } \\
\text { uniform mesh [37]. } \\
\text { Hp Finite Element Method [38]. } \\
\text { Terminal-boundary-value technique with uniform mesh [39]. } \\
\text { A hybrid initial value method with shishkin mesh [40]. } \\
\text { A fourth order finite difference scheme with uniform mesh [41]. } \\
\text { An Asymptotic-Numerical Hybrid Method [42]. }\end{array}$ \\
\hline
\end{tabular}

Table 1: Various Methods and Mesh used to solve SPDDE

Hp Finite Element Method: Serge et al. [38] used finite element method to find the solution of second order SPDDE.The solution of boundary value problem decomposed into smooth part, boundary layer part, an interior/interface layer part and a remainder. The method found to be of robustness and exponential rate of convergent.

Terminal boundary-value technique: In 2014, File et al. [39] solved the singularly perturbed delay differential equation of second order by using terminal boundary-value technique. A terminal point was introduced into the domain and the original problem is divided into inner and outer region problems. The terminal point introduced is common to the both inner and outer regions. The inner region problem is solved by choosing the transformation and the new
The method implemented on four numerical examples and found that presented method improved the findings of Soujanya and Reddy and Swamy. In this paper author discussed the effect of delay on amplitude of oscillations.

An Asymptotic-Numerical Hybrid Method: In 2017, Suleyman Cengizci [42] worked for approximations of the solutions of singularly perturbed second-order linear delay differential equation. The author considered following equation for numerical solution:

$\varepsilon y^{\prime \prime}(x)+p(x) y^{\prime}(x-\delta)+q(x) y(x)=r(x) \quad 0 \leq x \leq 1$ with $y(0)=\emptyset(x)$ on $\delta \leq x \leq 0$ and $y(1)=\gamma$.

Taylor series expansion used to linearized the delay term and resultant singularly perturbed ordinary differential equation 
solved by asymptotic method known as Successive complementary expansion method (SCEM). To test the scheme a left boundary layer problem considered for numerical simulation and the obtained solution was compared with exact solution and highly accurate approximations found in few iterations only.

The following table 1 shows various methods and mesh strategies used to solve SPDDE

\section{DISCUSSION AND CONCLUSION}

The study of Singularly Perturbed delay differential equation in the field of computational mathematics is very striking. The study of many theories and applied science results in singularly perturbed delay differential equation. Numerical solutions are important as they are easy and computed efficiently by using computer. The numerical and spline techniques appear to popular and ultimate tool to achieve the goal. This survey paper presented the development of various methods for different class of singularly perturbed delay differential equation. This paper will be very helpful to the researchers working in this area to develop the new numerical methods for solving singularly perturbed delay differential equations.

\section{References}

1. Kadalbajoo, Mohan K. and Reddy, Y. 1989. Asymptotic and numerical analysis of singular perturbation problems: a survey. Applied Mathematics and computation., 30(3): 223-259.

2. Kadalbajoo, Mohan K.andKailash C. Patidar. 2002. A survey of numerical techniques for solving singularly perturbed ordinary differential equations. Applied Mathematics and Computation., 130(2): 457-510.

3. Kadalbajoo, Mohan K.andKailash C. Patidar. 2003. Singularly perturbed problems in partial differential equations: a survey. Applied Mathematics and Computation., 134(2): 371-429.

4. Kadalbajoo, Mohan K. and Gupta, V. 2010. A brief survey on numerical methods for solving singularly perturbed problems. Applied Mathematics and Computation., 217(8): 3641-3716.

5. Sharma, Kapil K., Rai, P. and Kailash, C. P. 2013. A review on singularly perturbed differential equations with turning points and interior layers. Applied Mathematics and computation., 219(22): 10575-10609.

6. Lasota, A., and Wazewska, M. 1976. Mathematical models of the red blood cell system. Mat. Stos 6: 25-40.

7. Mackey, Michael C. and Glass,L . 1977. Oscillation and chaos in physiological control systems.Science 197(4300): 287-289.

8. Glizer, V.Y. 1998. Asymptotic solution of a singularly perturbed set of functional-differential equations of Riccati type encountered in the optimal control theory. Nonlinear Differential Equations and Applications NoDEA., 5(4): 491-515.

9. Glizer, V. Y. 2000. Asymptotic solution of a boundaryvalue problem for linear singularly-perturbed functional differential equations arising in optimal control theory.Journal of optimization theory and applications., 106(2): 309-335.
10. Assessment on veterinary voucher scheme in Rayitu, Guradamole and Dawe Qachen Woreda of Bale Lowlands

11.Lange, C.G. and Robert, M. M.1982.Singular perturbation analysis of boundary value problems for differential-difference equations.SIAM Journal on Applied Mathematics., 42(3): 502-531.

12.Stein, R B. 1967. Some models of neuronal variability.Biophysical journal., 7(1): 37

13. Ezzat, M. A., Mohamed, I.O. and Ahmed MS ElKaramany. 2002. State space approach to twodimensional generalized thermo-viscoelasticity with two relaxation times. International journal of engineering science., 40(11): 1251-1274.

14. Mallet-Paret, J. and Roger D.N. 1989. A differentialdelay equation arising in optics and physiology.SIAM Journal on Mathematical Analysis., 20(2): 249-292.

15. Aziz, T. and Khan, A. 2002. A spline method for second-order singularly perturbed boundary-value problems. Journal of Computational and Applied Mathematics., 147(2): 445-452.

16. Jha, N. 2012. Computational Method for Nonlinear Singularly Perturbed Singular Boundary Value Problems using Nonpolynomial Spline. Journal of Information and Computing Science., 7(2): 091-096

17. Abd El-Salam F. A. 2013. A parametric spline method for second-order singularly perturbed boundary-value problem.IOSR Journal of Mathematics (IOSR-JM)., 9(3): 01-05

18. Akram, G. and Naheed, A., 2013. Solution of fourth order singularly perturbed boundary value problem using septic spline. Middle-East Journal of Scientific Research., 15(2):302-311.

19.Doğan, N., Ertürk, V.S. and Akın, Ö. 2012. Numerical treatment of singularly perturbed two-point boundary value problems by using differential transformation method. Discrete Dynamics in Nature and Society.

20. Mishra, H.K. and Saini, S. 2013. Numerical solution of singularly perturbed two-point boundary value problem via liouville-green transform.

21.Gupta, Y. Srivastava, P.K. and Kumar, M. 2011. Application of B-Spline to Numerical Solution of a System of Singularly Perturbed Problems. Mathematica Aeterna., 1(6): 405-415.

22. Mishra, H.Kumar. 2015. Quartic B-Spline Method for Solving a Singular Singularly Perturbed Third-Order Boundary Value Problems. American Journal of Numerical Analysis.,3(1): 18-24.

23. Rihan, F.A. 2013. Delay Differential Equations in Biosciences: Parameter estimation and sensitivity analysis.Recent Advances in Applied Mathematics and Computational Methods: Proceedings of the 2013 International Conference on Applied Mathematics and Computational Methods (Venice, Italy September 2013).

24.Stein, R. B. 1967. Some models of neuronal variability.Biophysical journal.,7(1): 37.

25. Stein, R. B. 1965. A theoretical analysis of neuronal variability. Biophysical Journal., 5(2): 173.

26. Longtin, A. and Milton J.G.1988. Complex oscillations in the human pupil light reflex with "mixed" and delayed feedback.Mathematical Biosciences., 90(1-2): 183-199. 
27. Derstine, M. W., Gibbs, H. M., Hopf, F. A. and Kaplan, D. L. 1982. Bifurcation gap in a hybrid optically bistable system. Physical Review A., 26(6): 3720.

28. Bocharov, G A. and Rihan, F.A. 2000. Numerical modelling in biosciences using delay differential equations. Journal of Computational and Applied Mathematics., 125(1): 183-199.

29. Nelson, P.W. and Perelson, A.S. 2002. Mathematical analysis of delay differential equation models of HIV-1 infection.Mathematical biosciences., 179(1): 73-94.

30. Kumar, V. and Sharma, K.K. 2008. An optimized Bspline method for solving singularly perturbed differential difference equations with delay as well as advance. Neural, Parallel and Scientific Computations., 16(3): 371.

31.Kumar, D. and Kadalbajoo, M. K. 2012. Numerical Treatment of Singularly Perturbed Delay Differential Equations Using B-Spline Collocation Method on Shishkin Mesh. JNAIAM., 7(3)-4: 73-90.

32. Chakravarthy, P.P., Kumar, S.D., Rao, R.N. and Ghate, D.P. 2015. A fitted numerical scheme for second order singularly perturbed delay differential equations via cubic spline in compression. Advances in Difference Equations., 2015(1):300.

33. Yüzbaş1, Ş. and Sezer, M. 2013. Exponential collocation method for solutions of singularly perturbed delay differential equations., In Abstract and Applied Analysis (Vol. 2013). Hindawi Publishing Corporation.

34. File, G. and Reddy, YN. 2013. Computational method for solving singularly perturbed delay differential equations with negative shift. International Journal of Applied Science and Engineering., 11(1): 101-113.

35. Swamy, D.K., Phaneendra, K., BenerjiBabu, A. and Reddy, Y. N.2015. Computational method for singularly perturbed delay differential equations with twin layers or oscillatory behaviour. Ain Shams Engineering Journal., 6(1): 391-398.
36. Kumar, D. 2013. A computational technique for solving boundary value problem with two small parameters. Electronic Journal of Differential Equations., 2013(30): $1-10$.

37. Andargie, A. and Reddy Y.N. 2012. An asymptoticfitted method for solving singularly perturbed delay differential equations. Applied Mathematics, 3(08): 895.

38. Nicaise, S. and Xenophontos, C. 2013. Robust approximation of singularly perturbed delay differential equations by the $\mathrm{hp}$ finite element method. Computational Methods in Applied Mathe Matics., 13(1):21-37.

39. File, G. and Reddy, Y.N. 2014. Terminal boundaryvalue technique for solving singularly perturbed delay differential equations.Journal of Taibah University for Science., 8(3) : 289-300.

40. Subburayan, V. 2016. An hybrid initial value method for singularly perturbed delay differential equations with interior layers and weak boundary layer. Ain Shams Engineering Journal.

41.File, G., Gadisa, G., Aga, T. and Reddy Y.N. 2017. Numerical Solution of Singularly Perturbed Delay Reaction-Diffusion Equations with Layer or Oscillatory Behaviour. American Journal of Numerical Analysis., 5(1): $1-10$

42. Cengizci, S. 2017. An Asymptotic-Numerical Hybrid Method for Solving Singularly Perturbed Linear Delay Differential Equations. International Journal of Differential Equations.

\section{Please cite this article in press as:}

Mandeep Kaur and Geeta Arora (2017), A Review on Singular Perturbed Delay Differential Equations, International Journal of Current Advanced Research, 6(3), pp. 2341-2346.

http://dx.doi.org/10.24327/ijcar.2017. 2346.0005 